\title{
MAGNETIC MOMENTS OF HEAVY BARYONS IN THE BAG MODEL REEXAMINED
}

\author{
A. Bernotas ${ }^{a}$ and V. Šimonis ${ }^{b}$ \\ ${ }^{a}$ Faculty of Physics, Vilnius University, Sauletekio 9, LT-10222 Vilnius, Lithuania \\ b Institute of Theoretical Physics and Astronomy, Vilnius University, \\ A. Goštauto 12, LT-01108, Vilnius, Lithuania \\ E-mail: vytautas.simonis@tfai.vu.lt
}

Received 17 September 2012; accepted 20 June 2013

\begin{abstract}
The magnetic moments of $J=\frac{1}{2}$ and $J=\frac{3}{2}$ heavy baryons are calculated in the bag model with center-ofmass motion corrections. For the spin $\frac{1}{2}$ baryons containing three quarks of different flavours the effect of hyperfine mixing is examined in detail. The results of the work are compared with predictions obtained in various other approaches and models.
\end{abstract}

Keywords: bag model, magnetic moments, heavy baryons, state mixing

PACS: 12.39.Ba, 13.40.Em, 14.20.Lq, 14.20.Mr

\section{Introduction}

In recent years the interest in properties of heavy baryons has grown (for review see, e.g. [3]). A relevant source of information about the internal structure of particles is their magnetic moments. Magnetic moments of heavy baryons have been considered in various approaches. Nevertheless, the most extensive evaluation of these quantities remains the bag model calculations performed more than 30 years ago [2]. Sometimes these bag model predictions still may serve as a useful guide, yet several drawbacks are also evident. Firstly, only the ratios of magnetic moments to that of proton were presented. Absolute values in analogy with the case of light baryons were expected to be somewhat too small. Secondly, the list of ground state baryons used in Ref. [2] was incomplete - the states $\Xi_{b c}, \Xi_{b c}^{\prime}$ $\Omega_{b c}, \Omega_{b c}^{\prime}, \Omega_{b c c}$ and $\Omega_{b b c}$ were missing. Thirdly, the hyperfine mixing of $\Xi_{c}, \Xi_{c}^{\prime}$ states (as well as $\Xi_{b}, \Xi_{b}^{\prime}$ ) was not taken into account, which, as was shown in [3], can change the predictions for magnetic moments substantially. Finally, the parameters used in calculations of magnetic moments were chosen rather arbitrarily. The bag radii of charmed and bottom baryons were taken from different variants of the bag model (Ref. [4] and Ref. [5], respectively), and the mass of the charmed quark did not correspond to any of them. Therefore, the predictions of Ref. [2] for baryons containing charmed quarks cannot be treated as exact bag model result, but rather as a (more or less) crude estimate. We think that the contemporary update of the bag model predictions is necessary. In a recent paper [6] we have used an improved bag model to calculate the magnetic moments of light baryons. It was shown that the bag model with the center-of-mass motion (c.m.m.) corrections taken into account can provide sufficiently good predictions for magnetic moments. Now we are going to apply this model to calculate magnetic moments of $J=\frac{1}{2}$ and $J=\frac{3}{2}$ heavy baryons. 
The paper is structured as follows. In Sec. 2 we present briefly the model and give expressions for the baryon magnetic moments. In Sec. 3 we discuss quark model relations (sum rules) that connect magnetic moments of different baryons. Section 4 is devoted completely to the state mixing problem. Results of our calculations are presented in Sec. 5. The latter also serves for discussion and contains concluding remarks.

\section{Preliminaries: bag model, magnetic moments}

In our previous paper [6] two slightly different variants of the bag model suitable to provide a correct scale of the baryon magnetic moments were considered. In the present work we have chosen to deal with a first variant of these two because of its simplicity and universality. Below we will briefly outline the main features of this bag model variant.

The hadron bag energy depends on the bag radius $R$ and is given by

$$
E=\frac{4 \pi}{3} B R^{3}+\frac{Z_{0}}{R}+\sum_{i} \varepsilon_{i}+\Delta E,
$$

where the four terms in the expression are: the bag volume energy, the Casimir energy, the sum of single-particle eigenenergies, and the quark-quark interaction energy (for more details see [7]).

The interaction energy is defined to the first order in the effective (running) coupling constant

$$
\alpha_{\mathrm{c}}(R)=\frac{2 \pi}{9 \ln \left(A+R_{0} / R\right)},
$$

where $A$ and $R_{0}$ are model parameters. Up and down quarks are assumed to be massless. For heavier (strange, ${ }_{1}$ charmed, and bottom) quarks we use the running mass defined as

$$
\bar{m}_{f}(R)=\tilde{m}_{f}+\alpha_{\mathrm{c}}(R) \cdot \delta_{f},
$$

where $\widetilde{m}_{f}$ and $\delta_{f}$ are additional flavour-dependent parameters of the model.

The bag radius $R_{\mathrm{B}}$ of an individual hadron is obtained by minimizing (1) with respect to $R$. The mass of the hadron is related to the corresponding bag energy via expression

$$
M^{2}=E^{2}-P^{2},
$$

where the effective momentum square $P^{2}$ is defined as

$$
P^{2}=\gamma \sum_{i} p_{i}^{2}
$$

Here $p_{i}$ are the momenta of individual quarks, and the c.m.m. parameter $\gamma$ is to be determined in a fitting procedure.

The c.m.m. corrected magnetic moments are given by the Halprin and Kerman [8] relation

$$
\mu=\frac{E}{M} \mu^{0} .
$$

Altogether we have eleven free parameters in the model. These are: the bag constant $B$, the Casimir energy parameter $Z_{0}$, the c.m.m. parameter $\gamma$, two parameters from the definition of the running coupling constant ( $A$ and $R_{0}$ ), and six parameters necessary to define the running mass functions for the strange, charmed, and bottom quarks $\left(\widetilde{m}_{s}, \delta_{s} ; \widetilde{m}_{c}, \delta_{s}\right.$; $\left.\widetilde{m}_{b}, \delta_{b}\right)$. The values of $B, Z_{0}, \gamma, A, R_{0}, \tilde{m}_{s}, \delta_{s}$ were determined in Ref. [6]. They are: $B=7.468 \cdot 10^{-4} \mathrm{GeV}^{4}$, $Z_{0}=0.22, \gamma=2.153, A=0.6514, R_{0}=4.528 \mathrm{GeV}^{-1}$, $\tilde{m}_{s}=0.262 \mathrm{GeV}, \delta_{s}=0.083 \mathrm{GeV}$. The numerical values of the remaining four parameters $\widetilde{m}_{c}, \delta_{c}, \widetilde{m}_{b}$ and $\delta_{b}$ were obtained in the present work from the fit to the corresponding masses of $J / \psi, \Lambda_{c}, \Upsilon$, and $\Lambda_{b}$. They are: $\widetilde{m}_{c}=1.458 \mathrm{GeV}, \delta_{c}=0.089 \mathrm{GeV}$, $\widetilde{m}_{b}=4.721 \mathrm{GeV}$, and $\delta_{b}=0.079 \mathrm{GeV}$. As we see, the numerical values of parameter $\delta_{f}$ for all three flavours are similar. So, in principle, we could even reduce the number of free parameters of the model and use one average value (e.g., $\bar{\delta}=0.084 \mathrm{GeV}$ ) for strange, charmed, and bottom quarks.

The wave functions of baryons can be constructed by coupling the spins of the two first quarks to an intermediate spin $S$ and then adding the third one to form the baryon with the resulting spin $J$. Proceeding in such manner one can construct the so-called antisymmetric (with respect to the interchange of $q_{1}$ and $q_{2}$ ),

$$
\left|\left[q_{1} q_{2}\right] q_{3}\right\rangle=\left|\left(q_{1} q_{2}\right)^{S=0} q_{3}\right\rangle,
$$

and symmetric,

$$
\left|\left\{q_{1} q_{2}\right\} q_{3}\right\rangle=\left|\left(q_{1} q_{2}\right)^{S=1} q_{3}\right\rangle,
$$

states. For identically flavoured quarks $q_{1}$ and $q_{2}$ only the symmetric states are allowed. 
The valence quark contribution to the magnetic moments of baryons is given by [3, 9]

$$
\begin{aligned}
& S=0, J=\frac{1}{2} ; \quad \mu\left(\left[q_{1} q_{2}\right] q_{3}\right)=\mu_{3}, \\
& S=1, J=\frac{1}{2} ; \quad \mu\left(\left\{q_{1} q_{2}\right\} q_{3}\right)=\frac{1}{3}\left(2 \mu_{1}+2 \mu_{2}-\mu_{3}\right),
\end{aligned}
$$

and

$$
S=1, J=\frac{3}{2} ; \mu\left(q_{1} q_{2} q_{3}\right)=\mu_{1}+\mu_{2}+\mu_{3},
$$

where $\mu_{i}$ represent the magnetic moments of individual quarks. For the transition moments we have

$$
\begin{aligned}
& \mu^{\mathrm{tr}}\left(q_{1} q_{2} q_{3}\right)=\mu\left(\left\{q_{1} q_{2}\right\} q_{3} \rightarrow\left[q_{1} q_{2}\right] q_{3}\right) \\
& =\frac{1}{\sqrt{3}}\left(\mu_{2}-\mu_{1}\right) .
\end{aligned}
$$

In the bag model magnetic moments of confined quarks are given by the formula

$$
\mu_{i}=q_{i} \bar{\mu}_{i}
$$

where $q_{i}$ is the electric charge of the quark, and reduced (charge-independent) quark magnetic moments $\bar{\mu}_{i}$ depend on the quark mass $m_{i}$, energy $\varepsilon_{i}$, and bag radius of baryon $R_{B}$ (see [10]):

$$
\bar{\mu}_{i}=\frac{4 \varepsilon_{i} R_{\mathrm{B}}+2 m_{i} R_{\mathrm{B}}-3}{2\left(\varepsilon_{i} R_{\mathrm{B}}-1\right) \varepsilon_{i} R_{\mathrm{B}}+m_{i} R_{\mathrm{B}}} \frac{R_{\mathrm{B}}}{6} .
$$

The expression (12) changes its sign under the interchange of the first two quarks in the wave functions (7), (8). This is important in the sense that relative signs of transition moments are observable in an interference between decays to the same state. Therefore, the same quark ordering must be used consistently for each state of the same quarks. Of course, we are free to choose the relative phase of the wave function, but only once. Our phase conventions are the same as in Ref. [3] and differ from those adopted in e. g. Ref. [9].

For the baryons containing three quarks of different flavours the intermediate spins, in general, are not good quantum numbers because the colour-magnetic interaction causes the mixing of the states (7) and (8). This problem will be discussed in detail in Sec. 4.

Using Eqs. (9)-(12) one can write down the explicit expressions for the magnetic moments of all ground state heavy baryons. Sometimes it is useful to have such expressions at hand. For convenience we present them in the Appendix.

\section{Quark model relations and sum rules}

With some additional assumptions plenty of quark model relations between magnetic moments of various baryons can be obtained. The main assumption is that quark magnetic moments in various baryons are the same. This is true for the naive nonrelativistic quark model. In Ref. [9] this assumption was used to obtain the relations connecting magnetic moments of differently flavoured baryons (charmed and light, for example). Our opinion is that there must be no illusions about the predictive power of such relations.

In the bag model, the quark magnetic moments can differ when passing from baryon to baryon. Therefore, the before mentioned assumption may be treated as sufficiently accurate approximation only for baryons with the same (or very similar) quark content. In other cases one should handle it with care.

From the expressions presented in the columns 3 of Tables 8 and 10 (in the Appendix) we readily obtain the relations for magnetic moments of charmed baryons:

$$
\begin{aligned}
& \mu\left(\Sigma_{c}^{++}\right)+\mu\left(\Sigma_{c}^{0}\right)=2 \mu\left(\Sigma_{c}^{+}\right) \\
& \mu\left(\Sigma_{c}^{*++}\right)+\mu\left(\Sigma_{c}^{* 0}\right)=2 \mu\left(\Sigma_{c}^{*+}\right), \\
& \mu\left(\Lambda_{c}^{+}\right)+\mu\left(\Sigma_{c}^{+}\right)=\frac{2}{3} \mu\left(\Sigma_{c}^{*+}\right), \\
& \mu\left(\Xi_{c}^{0}\right)+\mu\left(\Xi_{c}^{\prime 0}\right)=\frac{2}{3} \mu\left(\Xi_{c}^{* 0}\right), \\
& \mu\left(\Xi_{c}^{+}\right)+\mu\left(\Xi_{c}^{++}\right)=\frac{2}{3} \mu\left(\Xi_{c}^{*+}\right), \\
& \mu\left(\Sigma_{c}^{*++}\right)+\mu\left(\Omega_{c}^{* 0}\right)=2 \mu\left(\Xi_{c}^{*+}\right), \\
& 4 \mu\left(\Omega_{c c}^{+}\right)+\mu\left(\Omega_{c}^{0}\right) \approx 5 \mu\left(\Lambda_{c}^{+}\right), \\
& 2 \mu\left(\Omega_{c c}^{*+}\right)-\mu\left(\Omega_{c}^{* 0}\right) \approx 3 \mu\left(\Lambda_{c}^{+}\right), \\
& \mu\left(\Omega_{c c c}^{++}\right) \approx 3 \mu\left(\Lambda_{c}^{+}\right), \\
& 3\left[4 \mu\left(\Omega_{c}^{0}\right)+\mu\left(\Omega_{c c}^{+}\right)\right]=5\left[2 \mu\left(\Omega_{c}^{* 0}\right)-\mu\left(\Omega_{c c}^{*+}\right)\right], \\
& 3\left[4 \mu\left(\Omega_{c c}^{+}\right)+\mu\left(\Omega_{c}^{0}\right)\right] \approx 5\left[2 \mu\left(\Omega_{c c}^{*+}\right)-\mu\left(\Omega_{c}^{* 0}\right)\right] .
\end{aligned}
$$


In the naive nonrelativistic model there exist also relations $4 \mu\left(\Omega_{c}^{0}\right)+\mu\left(\Omega_{c c}^{+}\right)=5 \mu(\Lambda)$ and $2 \mu\left(\Omega^{*_{0}}\right)-\mu\left(\Omega_{c c}^{*_{+}}\right)=3 \mu(\Lambda)$, where $\mu(\Lambda)$ represents the magnetic moment of the strange baryon $\Lambda$. In the bag model, these relations do not hold anymore because magnetic moments of strange quarks entering light and heavy baryons differ substantially. However, we can combine these equations to obtain the first row of Eqs. (20). In order to distinguish between sufficiently accurate (in our model) and approximate relations we use the symbol " = " in the cases when the accuracy of the relation is $\leq 3 \%$, and the symbol " $\approx$ " when the accuracy is in the range (4-9)\%. If the accuracy is worse, the relation is discarded.

An isospin symmetry leads to additional relations. For the magnetic moments this symmetry means that one can set $\bar{\mu}_{d}=\bar{\mu}_{d}$. Then from columns 4 of Tables 8 and 10 the following relations can be deduced:

$$
\begin{aligned}
& \mu\left(\Sigma_{c}^{* 0}\right)=-3 \mu\left(\Sigma_{c}^{+}\right), \\
& \mu\left(\Sigma_{c}^{*++}\right)=-3 \mu\left(\Sigma_{c}^{0}\right), \\
& 2 \mu\left(\Sigma_{c}^{+}\right)+\mu\left(\Sigma_{c}^{0}\right)=-\mu\left(\Lambda_{c}^{+}\right), \\
& \mu\left(\Sigma_{c}^{*+}\right)-\mu\left(\Sigma_{c}^{* 0}\right)=\mu\left(\Sigma_{c}^{*++}\right)-\mu\left(\Sigma_{c}^{*+}\right)= \\
& \mu\left(\Xi_{c}^{*+}\right)-\mu\left(\Xi_{c}^{* 0}\right)=-\left[\mu\left(\Sigma_{c}^{+}\right)-\mu\left(\Sigma_{c}^{0}\right)\right], \\
& 2 \mu\left(\Xi_{c c}^{+}\right)+\mu\left(\Xi_{c c}^{++}\right)=\frac{4}{3} \mu\left(\Omega_{c c}^{++}\right), \\
& 2 \mu\left(\Xi_{c c}^{*+}\right)+\mu\left(\Xi_{c c}^{*++}\right)=2 \mu\left(\Omega_{c c c}^{++}\right), \\
& 2 \mu\left(\Sigma_{c}^{*+}\right)+\mu\left(\Sigma_{c}^{* 0}\right)=3 \mu\left(\Lambda_{c}^{+}\right), \\
& \mu\left(\Xi_{c c}^{*++}\right)-\mu\left(\Xi_{c c}^{*+}\right)=3\left[\mu\left(\Xi_{c c}^{+}\right)-\mu\left(\Xi_{c c}^{++}\right)\right], \\
& \mu\left(\Xi_{c c}^{*+}\right) \approx \frac{3}{2} \mu\left(\Xi_{c c}^{++}\right), \\
& \mu\left(\Xi_{c c}^{*++}\right) \approx 2 \mu\left(\Sigma_{c}^{*+}\right), \\
& \mu\left(\Xi_{c c}^{*++}\right)-\mu\left(\Xi_{c c}^{*+}\right) \approx \mu\left(\Xi_{c}^{*+}\right)-\mu\left(\Xi_{c}^{* 0}\right) .
\end{aligned}
$$

In the bottom sector the analogy of Eqs. (15)(20) is

$$
\begin{aligned}
& \mu\left(\Sigma_{b}^{+}\right)+\mu\left(\Sigma_{b}^{-}\right)=2 \mu\left(\Sigma_{b}^{0}\right), \\
& \mu\left(\Sigma_{b}^{*+}\right)+\mu\left(\Sigma_{b}^{*-}\right)=2 \mu\left(\Sigma_{b}^{* 0}\right),
\end{aligned}
$$

$$
\begin{aligned}
& \mu\left(\Lambda_{b}^{0}\right)+\mu\left(\Sigma_{b}^{0}\right)=\frac{2}{3} \mu\left(\Sigma_{b}^{* 0}\right), \\
& \mu\left(\Xi_{b}^{-}\right)+\mu\left(\Xi_{b}^{\prime-}\right)=\frac{2}{3} \mu\left(\Xi_{b}^{*-}\right), \\
& \mu\left(\Xi_{b}^{0}\right)+\mu\left(\Xi_{b}^{\prime 0}\right)=\frac{2}{3} \mu\left(\Xi_{b}^{* 0}\right), \\
& \mu\left(\Xi_{b c}^{0}\right)+\mu\left(\Xi_{b c}^{\prime 0}\right)=\frac{2}{3} \mu\left(\Xi_{b c}^{* 0}\right), \\
& \mu\left(\Xi_{b c}^{+}\right)+\mu\left(\Xi_{b c}^{\prime+}\right)=\frac{2}{3} \mu\left(\Xi_{b c}^{*+}\right), \\
& \mu\left(\Omega_{b c}^{0}\right)+\mu\left(\Omega_{b c}^{\prime 0}\right)=\frac{2}{3} \mu\left(\Omega_{b c}^{* 0}\right), \\
& \mu\left(\Sigma_{b}^{*+}\right)+\mu\left(\Omega_{b}^{*-}\right)=2 \mu\left(\Xi_{b}^{* 0}\right), \\
& \mu\left(\Omega_{b b b}^{-}\right)=3 \mu\left(\Lambda_{b}^{0}\right) . \\
& 4 \mu\left(\Omega_{b b c}^{0}\right)+\mu\left(\Omega_{b c c}^{+}\right) \approx 5 \mu\left(\Lambda_{b}^{0}\right), \\
& 2 \mu\left(\Omega_{b c c}^{*+}\right)-\mu\left(\Omega_{b b c}^{* 0}\right) \approx \mu\left(\Omega_{c c c}^{++}\right), \\
& 2 \mu\left(\Omega_{b b c}^{* 0}\right)-\mu\left(\Omega_{b c c}^{*+}\right) \approx \mu\left(\Omega_{b b b}^{-}\right), \\
& 3\left[4 \mu\left(\Omega_{b b c}^{0}\right)+\mu\left(\Omega_{b c c}^{+}\right)\right]=5\left[2 \mu\left(\Omega_{b b c}^{* 0}\right)-\mu\left(\Omega_{b c c}^{*+}\right)\right], \\
& 3\left[4 \mu\left(\Omega_{b c c}^{+}\right)+\mu\left(\Omega_{b b c}^{0}\right)\right]=5\left[2 \mu\left(\Omega_{b c c}^{*+}\right)-\mu\left(\Omega_{b b c}^{* 0}\right)\right], \\
& 3\left[4 \mu\left(\Omega_{b}^{-}\right)+\mu\left(\Omega_{b b}^{-}\right)\right]=5\left[2 \mu\left(\Omega_{b}^{*-}\right)-\mu\left(\Omega_{b b}^{*-}\right)\right] .
\end{aligned}
$$

In this case we have a problem with the quark model relations $4 \mu\left(\Omega_{b b}^{-}\right)+\mu\left(\Omega_{b}^{-}\right)=5 \mu\left(\Lambda_{b}^{0}\right)$ and $2 \mu$ $\left(\Omega_{b b}^{*-}\right)-\mu\left(\Omega_{b}^{*-}\right)=3 \mu\left(\Lambda_{b}^{0}\right)$. In the bag model both of them fail badly, and so does their combination $3\left[4 \mu\left(\Omega_{b b}^{-}\right)+\mu\left(\Omega_{b}^{-}\right)=5\left[2 \mu\left(\Omega_{b b}^{*-}\right)-\mu\left(\Omega_{b}^{*-}\right)\right]\right.$. The culprit is the strange quarks. The difference of their magnetic moments in the $\Omega_{b b}^{-}$and $\Omega_{b}^{-}$baryons is comparable with the magnetic moment of the bottom quark. As a consequence, the sufficiently accurate value of the latter cannot be extracted from the above relations, and we are forced to exclude them from our list.

The isospin symmetry now leads to the relations

$$
\begin{aligned}
& \mu\left(\Sigma_{b}^{*-}\right)=-3 \mu\left(\Sigma_{b}^{0}\right), \\
& \mu\left(\Sigma_{b}^{*+}\right)=-3 \mu\left(\Sigma_{b}^{-}\right), \\
& \mu\left(\Xi_{b b}^{*-}\right)=\frac{3}{2} \mu\left(\Xi_{b b}^{0}\right), \\
& \mu\left(\Sigma_{b}^{*+}\right)-\mu\left(\Sigma_{b}^{* 0}\right)=\mu\left(\Sigma_{b}^{* 0}\right)-\mu\left(\Sigma_{b}^{*-}\right)= \\
& \mu\left(\Xi_{b}^{* 0}\right)-\mu\left(\Xi_{b}^{*-}\right), \\
& \mu\left(\Xi_{b b}^{* 0}\right)-\mu\left(\Xi_{b b}^{*-}\right)=3\left[\mu\left(\Xi_{b b}^{-}\right)-\mu\left(\Xi_{b b}^{0}\right)\right], \\
& 2 \mu\left(\Xi_{b b}^{*-}\right)+\mu\left(\Xi_{b b}^{* 0}\right)=2 \mu\left(\Omega_{b b b}^{-}\right), \\
& 2 \mu\left(\Xi_{b b}^{-}\right)+\mu\left(\Xi_{b b}^{0}\right)=4 \mu\left(\Lambda_{b}^{0}\right),
\end{aligned}
$$




$$
\begin{aligned}
& \mu\left(\Xi_{b b}^{* 0}\right) \approx 2 \mu\left(\Sigma_{b}^{* 0}\right), \\
& \mu\left(\Xi_{b c}^{*+}\right)-\mu\left(\Xi_{b c}^{* 0}\right) \approx \mu\left(\Xi_{b}^{* 0}\right)-\mu\left(\Xi_{b}^{*-}\right) .
\end{aligned}
$$

The states of the type $B, B^{\prime}$ enter the relations above only in the combination $\mu(B)+\mu\left(B^{\prime}\right)$. The reason is the state mixing which can cause sizable shifts of unmixed quantities while leaving, however, the combination $\mu(B)+\mu\left(B^{\prime}\right)$ invariant. There are several states the mixing of which is sufficiently small. First of all, such are two pairs of states $\Lambda_{c}^{+}, \Sigma_{c}^{+}$ and $\Lambda_{b}^{0}, \Sigma_{b}^{0}$, for which in the case of exact isospin symmetry there is not any mixing at all. A careful analysis [3] shows that this assumption is valid to a high degree also for real physical states. Therefore, the mixing between $\Lambda_{Q}$ and $\Sigma_{Q}$ states (where $Q$ denotes heavy quark) can be safely ignored. Explicit calculations show that in the bag model the mixing of $\Xi_{c}^{0}, \Xi_{c}^{\prime 0}$ (as well as $\Xi_{b}^{-}, \Xi_{b}^{\prime-}$ ) is not large enough to change the unmixed magnetic moments substantially. Therefore, we can add two more relations to our collection:

$$
\begin{aligned}
& \mu\left(\Xi_{c}^{0}\right) \approx \mu\left(\Lambda_{c}^{+}\right), \\
& \mu\left(\Xi_{b}^{-}\right) \approx \mu\left(\Lambda_{b}^{0}\right) .
\end{aligned}
$$

That is all that remains from the naive nonrelativistic result

$$
\begin{aligned}
& \mu\left(\Lambda_{c}^{+}\right)=\mu\left(\Xi_{c}^{0}\right)=\mu\left(\Xi_{c}^{+}\right), \\
& \mu\left(\Lambda_{b}^{0}\right)=\mu\left(\Xi_{b}^{0}\right)=\mu\left(\Xi_{b}^{-}\right)=\mu\left(\Xi_{b c}^{0}\right)=\mu\left(\Xi_{b c}^{+}\right)=\mu\left(\Omega_{b c}^{0}\right) .
\end{aligned}
$$

One may wonder why one member of the isospin doublet (i.e. $\Xi_{c}^{+}$) undergoes substantial changes, but another $\left(\Xi_{c}^{0}\right)$ does not. The reason is a very large $\Xi_{c}^{\prime+} \rightarrow \Xi_{c}^{+}$transition magnetic moment, larger by an order of magnitude than the corresponding $\Xi_{c}^{\prime 0} \rightarrow \Xi_{c}^{0}$ moment. The same is also true for the isospin doublet $\Xi_{b}^{-}, \Xi_{b}^{0}$.

A reasonable question is if there could be any profit from all these nice relations and sum rules. Of course, they can be used as a tool for the extra check of the results obtained in calculations. They may help one to gain some feeling (plausibly oversimplified) about the possible values of magnetic moments under consideration and reveal some regularities as well. It is not clear in general if many of them would survive in other approaches, especially the more elaborated ones with various corrections included. In the heavy hadron chiral perturbation theory [11], for example, Eqs. (15), (17), (22), (26), (28), (33) are valid.
In the end, we think that caution must be paid while trying to use a single magnetic moment of baryons $\Xi_{c}^{+}, \Xi_{c}^{\prime+}, \Xi_{b}^{0}, \Xi_{b}^{\prime 0}, \Xi_{b c}^{0}, \Xi_{b c}^{\prime 0}, \Xi_{b c}^{+}, \Xi_{b c}^{\prime+}, \Omega_{b c}^{0}$ and $\Omega_{b c}^{\prime 0}$ in the quark model sum rules such as, for example, $\mu\left(\Sigma_{c}^{++}\right)+\mu\left(\Omega_{c}^{0}\right)=2 \mu\left(\Xi_{c}^{\prime+}\right)[11$. State mixing effect can spoil this relation. In our model its accuracy is only about $25 \%$. The usual relations containing these states are valid only when the unmixed states are considered. In the case of physical states only the invariant combination of the type $\mu(B)+\mu\left(B^{\prime}\right)$ makes sense.

\section{Wave function mixing}

Among the heavy baryons under consideration there are some containing three quarks of different flavours. In this case additional complications arise because the quark-quark hyperfine interaction is not diagonal in the basis defined by the wave functions (7), (8). The physical states are the linear combinations of these states:

$$
\begin{aligned}
& |B\rangle=C_{1}\left|\left[q_{1} q_{2}\right] q_{3}\right\rangle+C_{2}\left|\left\{q_{1} q_{2}\right\} q_{3}\right\rangle, \\
& \left|B^{\prime}\right\rangle=-C_{2}\left|\left[q_{1} q_{2}\right] q_{3}\right\rangle+C_{1}\left|\left\{q_{1} q_{2}\right\} q_{3}\right\rangle .
\end{aligned}
$$

We have already studied the impact of such state mixing on the masses of heavy baryons in Ref. [12]. An extensive study was also performed in the framework of a nonrelativistic potential model [13, 14, and it was shown that the state mixing has significant implications for some aspects of phenomenology of these states such as their semileptonic decay rates. In Ref. [3] it was shown that this mixing can affect the values of magnetic moments even when it is not sufficiently strong to induce significant shifts of baryon masses.

When the state mixing is taken into account the mixed magnetic moments of the baryons are given by

$$
\begin{aligned}
& \mu(B)=C_{1}^{2} \mu\left(\left[q_{1} q_{2}\right] q_{3}\right)+C_{2}^{2} \mu\left(\left\{q_{1} q_{2}\right\} q_{3}\right)+ \\
& 2 C_{1} C_{2} \mu^{\mathrm{tr}}\left(q_{1} q_{2} q_{3}\right), \\
& \mu\left(B^{\prime}\right)=C_{1}^{2} \mu\left(\left\{q_{1} q_{2}\right\} q_{3}\right)+C_{2}^{2} \mu\left(\left[q_{1} q_{2}\right] q_{3}\right)- \\
& 2 C_{1} C_{2} \mu^{\mathrm{tr}}\left(q_{1} q_{2} q_{3}\right) .
\end{aligned}
$$

The physical transition moment is now

$$
\begin{aligned}
& \mu\left(B^{\prime} \rightarrow B\right)=\left(C_{1}^{2}-C_{2}^{2}\right) \mu^{\mathrm{tr}}\left(q_{1} q_{2} q_{3}\right)+ \\
& C_{1} C_{2}\left[\mu\left(\left\{q_{1} q_{2}\right\} q_{3}\right)-\mu\left(\left[q_{1} q_{2}\right] q_{3}\right)\right] .
\end{aligned}
$$


Table 1. Mixed and unmixed magnetic moments (in nuclear magnetons) of $\Xi_{c}, \Xi_{c}^{\prime}$, and $\Xi_{b}, \Xi_{b}^{\prime}$ baryons.

\begin{tabular}{|c|c|c|c|c|c|}
\hline Wave function & $\begin{array}{l}C_{1} \\
C_{2} \\
\end{array}$ & $\begin{array}{l}\text { Magnetic } \\
\text { moments }\end{array}$ & Wave function & $\begin{array}{l}C_{1} \\
C_{2} \\
\end{array}$ & $\begin{array}{l}\text { Magnetic } \\
\text { moments }\end{array}$ \\
\hline$\left\{\begin{array}{c}\left|\Xi_{c}^{0}\right\rangle \\
\left|\Xi_{c}^{\prime 0}\right\rangle \\
\left|\Xi_{c}^{\prime 0}\right\rangle \rightarrow\left|\Xi_{c}^{0}\right\rangle\end{array}\right.$ & & $\begin{array}{c}0.421 \\
-0.914 \\
0.013\end{array}$ & $\left\{\begin{array}{c}\left|\Xi_{c}^{+}\right\rangle \\
\left|\Xi_{c}^{\prime+}\right\rangle \\
\left|\Xi_{c}^{\prime+}\right\rangle \rightarrow\left|\Xi_{c}^{+}\right\rangle\end{array}\right.$ & & $\begin{array}{c}0.257 \\
0.591 \\
-1.043\end{array}$ \\
\hline$\left\{\begin{array}{c}|[d s] c\rangle \\
|\{d s\} c\rangle \\
|\{d s\} c\rangle \rightarrow|[d s] c\rangle\end{array}\right.$ & $\begin{array}{l}0.997 \\
0.073 \\
\ldots\end{array}$ & $\begin{array}{c}0.412 \\
-0.905 \\
0.110\end{array}$ & $\begin{array}{c}|[u s] c\rangle \\
|\{u s\} c\rangle \\
\{u s\} c\rangle \rightarrow|[u s] c\rangle\end{array}$ & $\begin{array}{c}0.997 \\
-0.073 \\
\ldots\end{array}$ & $\begin{array}{c}0.412 \\
0.438 \\
-1.057\end{array}$ \\
\hline$\left\{\begin{array}{c}|[c d] s\rangle \\
|\{c d\} s\rangle \\
|\{c d\} s\rangle \rightarrow|[c d] s\rangle\end{array}\right.$ & $\begin{array}{c}-0.562 \\
0.827 \\
\ldots\end{array}$ & $\begin{array}{l}-0.482 \\
-0.015 \\
-0.626\end{array}$ & $\left\{\begin{array}{c}|[c u] s\rangle \\
|\{c u\} s\rangle \\
|\{c u\} s\rangle \rightarrow|[c u] s\rangle\end{array}\right.$ & $\begin{array}{c}-0.562 \\
0.827 \\
\ldots\end{array}$ & $\begin{array}{c}-0.482 \\
1.335 \\
0.541\end{array}$ \\
\hline$\left\{\begin{array}{c}|[s c] d\rangle \\
|\{s c\} d\rangle \\
|\{s c\} d\rangle \rightarrow|[s c] d\rangle\end{array}\right.$ & $\begin{array}{c}-0.435 \\
-0.900 \\
\ldots\end{array}$ & $\begin{array}{c}-0.672 \\
0.177 \\
0.516\end{array}$ & $\left\{\begin{array}{c}|[s c] u\rangle \\
|\{s c\} u\rangle \\
|\{s c\} u\rangle \rightarrow|[s c] u\rangle\end{array}\right.$ & $\begin{array}{c}-0.435 \\
-0.900 \\
\ldots\end{array}$ & $\begin{array}{c}1.344 \\
-0.498 \\
0.516\end{array}$ \\
\hline$\left\{\begin{array}{c}\left|\Xi_{b}^{-}\right\rangle \\
\left|\Xi_{b}^{-}\right\rangle \\
\left|\Xi_{b}^{\prime-}\right\rangle \rightarrow\left|\Xi_{b}^{-}\right\rangle\end{array}\right.$ & & $\begin{array}{c}-0.063 \\
-0.660 \\
0.082\end{array}$ & $\left\{\begin{array}{c}\left|\Xi_{b}^{0}\right\rangle \\
\left|\Xi_{b}^{\prime 0}\right\rangle \\
\left|\Xi_{b}^{\prime 0}\right\rangle \rightarrow\left|\Xi_{b}^{0}\right\rangle\end{array}\right.$ & & $\begin{array}{c}-0.100 \\
0.556 \\
-0.917\end{array}$ \\
\hline$\left\{\begin{array}{c}|[d s] b\rangle \\
|\{d s\} b\rangle \\
|\{d s\} b\rangle \rightarrow|[d s] b\rangle\end{array}\right.$ & $\begin{array}{c}0.999 \\
0.018 \\
\quad \ldots\end{array}$ & $\begin{array}{c}-0.066 \\
-0.656 \\
0.093\end{array}$ & $\left\{\begin{array}{c}|[u s] b\rangle \\
|\{u s\} b\rangle \\
|\{u s\} b\rangle \rightarrow|[u s] b\rangle\end{array}\right.$ & $\begin{array}{l}0.999 \\
0.018 \\
\quad \ldots\end{array}$ & $\begin{array}{c}-0.066 \\
0.522 \\
-0.928\end{array}$ \\
\hline$\left\{\begin{array}{c}|[b d] s\rangle \\
|\{b d\} s\rangle \\
|\langle b d\} s\rangle \rightarrow|[b d] s\rangle\end{array}\right.$ & $\begin{array}{c}-0.516 \\
0.857 \\
\ldots\end{array}$ & $\begin{array}{l}-0.428 \\
-0.294 \\
-0.302\end{array}$ & $\left\{\begin{array}{c}|[b u] s\rangle \\
|\{b u\} s\rangle \\
|\{b u\} s\rangle \rightarrow|[b u] s\rangle\end{array}\right.$ & $\begin{array}{c}-0.516 \\
0.857 \\
\ldots\end{array}$ & $\begin{array}{c}-0.428 \\
0.885 \\
0.719\end{array}$ \\
\hline$\left\{\begin{array}{c}|[s b] d\rangle \\
|\{s b\} d\rangle \\
|\{s b\} d\rangle \rightarrow|[s b] d\rangle\end{array}\right.$ & $\begin{array}{c}-0.484 \\
-0.875 \\
\ldots\end{array}$ & $\begin{array}{c}-0.589 \\
-0.133 \\
0.209\end{array}$ & $\left\{\begin{array}{c}|[s b] u\rangle \\
|\{s b\} u\rangle \\
|\{s b\} u\rangle \rightarrow|[s b] u\rangle\end{array}\right.$ & $\begin{array}{c}-0.448 \\
-0.875 \\
\ldots\end{array}$ & $\begin{array}{c}1.179 \\
-0.723 \\
0.209\end{array}$ \\
\hline
\end{tabular}

Without mixing the results in most cases depend very strongly on the quark ordering in the spin coupling scheme $\left[\left(q_{1} q_{2}\right)^{s} q_{3}\right]^{J}$. With mixing the quark ordering becomes irrelevant, and in every case the final result is the same. Various authors use in their calculations different quark arrangements (very often with no state mixing taken into account), and therefore sometimes it is not obvious how to compare our results with other ones. To make things clearer, we present in Tables 1, 2 the results of our calculations of heavy baryon magnetic moments with intermediate data for all possible quark orderings. We think it is a good pedagogi- cal example, too. The calculation procedure is almost the same as in Ref. [12]. The only difference is the opposite sign of the off-diagonal matrix element of the interaction energy. We have changed the relative phase of the wave functions in order to have the same phase conventions as in Ref. [3]. The quark arrangements used are: $\left(q_{1} q_{2}\right)^{S} q_{3},\left(q_{3} q_{1}\right)^{S} q_{2}$, and $\left(q_{2} q_{3}\right)^{S} q_{1}$, where in the first one the quarks are ordered from lightest to heaviest. Note that in order to maintain the relative phases of wave functions unchanged the second and the third schemes are obtained from the first one by even permutation of particles. Coefficients $C_{1}, C_{2}$ in Tables 1, 2 
Table 2. Mixed and unmixed magnetic moments (in nuclear magnetons) of doubly heavy baryons $\Xi_{b c}$, $\Xi_{b c}^{\prime}$, and $\Omega_{b c}$, $\Omega_{b c}^{\prime}$.

\begin{tabular}{|c|c|c|c|c|c|}
\hline Wave function & $\begin{array}{l}C_{1} \\
C_{2}\end{array}$ & $\begin{array}{l}\text { Magnetic } \\
\text { moments }\end{array}$ & Wave function & $\begin{array}{l}C_{1} \\
C_{2}\end{array}$ & $\begin{array}{l}\text { Magnetic } \\
\text { moments }\end{array}$ \\
\hline$\left\{\begin{array}{c}\left|\Xi_{b c}^{0}\right\rangle \\
\left|\Xi_{b c}^{\prime 0}\right\rangle \\
\left|\Xi_{b c}^{\prime 0}\right\rangle \rightarrow\left|\Xi_{b c}^{0}\right\rangle\end{array}\right.$ & & $\begin{array}{c}0.068 \\
-0.236 \\
0.508\end{array}$ & 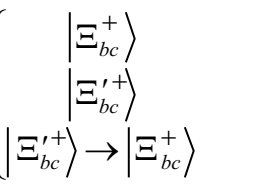 & & $\begin{array}{c}-0.157 \\
1.093 \\
-0.277\end{array}$ \\
\hline$\left\{\begin{array}{c}|[d c] b\rangle \\
|\{d c\} b\rangle \\
|\{d c\} b\rangle \rightarrow|[d c] b\rangle\end{array}\right.$ & $\begin{array}{c}0.992 \\
0.128 \\
\ldots\end{array}$ & $\begin{array}{c}-0.066 \\
-0.102 \\
0.530\end{array}$ & $\begin{array}{c}|[u c] b\rangle \\
|\{u c\} b\rangle \\
|\{u c\} b\rangle \rightarrow|[u c] b\rangle\end{array}$ & $\begin{array}{c}0.992 \\
0.128 \\
\ldots\end{array}$ & $\begin{array}{c}-0.066 \\
1.002 \\
-0.427\end{array}$ \\
\hline$\left\{\begin{array}{c}|[b d] c\rangle \\
|\{b d\} c\rangle \\
|\langle b d\} c\rangle \rightarrow|[b d] c\rangle\end{array}\right.$ & $\begin{array}{c}-0.607 \\
0.795 \\
\ldots\end{array}$ & $\begin{array}{c}0.366 \\
-0.534 \\
-0.281\end{array}$ & $\left\{\begin{array}{c}|[b u] c\rangle \\
|\{b u\} c\rangle \\
|\{b u\} c\rangle \rightarrow|[b u] c\rangle\end{array}\right.$ & $\begin{array}{c}-0.607 \\
0.795 \\
\ldots\end{array}$ & $\begin{array}{l}0.366 \\
0.571 \\
0.676\end{array}$ \\
\hline$\left\{\begin{array}{c}|[c b] d\rangle \\
|\{c b\} d\rangle \\
|\{c b\} d\rangle \rightarrow|[c b] d\rangle\end{array}\right.$ & $\begin{array}{c}-0.385 \\
-0.923 \\
\ldots\end{array}$ & $\begin{array}{c}-0.552 \\
0.384 \\
-0.249\end{array}$ & $\begin{array}{c}|[c b] u\rangle \\
|\{c b\} u\rangle \\
|\{c b\} u\rangle \rightarrow|[c b] u\rangle\end{array}$ & $\begin{array}{c}-0.385 \\
-0.923 \\
\ldots\end{array}$ & $\begin{array}{c}1.105 \\
-0.168 \\
-0.249\end{array}$ \\
\hline$\left\{\begin{array}{c}\left|\Omega_{b c}^{0}\right\rangle \\
\left|\Omega_{b c}^{\prime 0}\right\rangle \\
\left|\Omega_{b c}^{\prime 0}\right\rangle \rightarrow\left|\Omega_{b c}^{0}\right\rangle\end{array}\right.$ & & $\begin{array}{c}0.034 \\
-0.106 \\
0.443\end{array}$ & & & \\
\hline$\left\{\begin{array}{c}|[s c] b\rangle \\
|\{s c\} b\rangle \\
|\{s c\} b\rangle \rightarrow|[s c] b\rangle\end{array}\right.$ & $\begin{array}{c}0.994 \\
0.112 \\
\ldots\end{array}$ & $\begin{array}{c}-0.066 \\
-0.007 \\
0.447\end{array}$ & & & \\
\hline$\left\{\begin{array}{c}|[b s] c\rangle \\
|\{b s\} c\rangle \\
|\langle b s\} c\rangle \rightarrow|[b s] c\rangle\end{array}\right.$ & $\begin{array}{c}-0.593 \\
0.805 \\
\ldots\end{array}$ & $\begin{array}{c}0.366 \\
-0.440 \\
-0.198\end{array}$ & & & \\
\hline$\left\{\begin{array}{c}|[c b] s\rangle \\
|\{c b\} s\rangle \\
|\{c b\} s\rangle \rightarrow|[c b] s\rangle\end{array}\right.$ & $\begin{array}{c}-0.400 \\
-0.916 \\
\ldots\end{array}$ & $\begin{array}{c}-0.409 \\
0.336 \\
-0.249\end{array}$ & & & \\
\hline
\end{tabular}

define the expansion of the mixed state $|B\rangle=C_{1}\left|\left[q_{1} q_{2}\right] q_{3}\right\rangle+C_{2}\left|\left\{q_{1} q_{2}\right\} q_{3}\right\rangle$.

From the inspection of Tables 1 and 2 it is clear that unmixed magnetic moments are very sensitive to the quark ordering scheme, and therefore, strictly speaking, for magnetic moments there is no good ordering scheme. For the states $\Xi_{c}^{0}, \Xi_{c}^{\prime 0}$ and $\Xi_{b}^{-}, \Xi_{b}^{\prime-}$ the basis with the heaviest quark standing in the end [3, 12] still remains preferable, but for other states $\left(\Xi_{c}^{+}, \Xi_{c}^{\prime+} ; \Xi_{b}^{0}, \Xi_{b}^{\prime 0} ; \Xi_{b c}^{0}, \Xi_{b c}^{\prime 0} ; \Xi_{b c}^{+}, \Xi_{b c}^{\prime+}\right.$; and $\left.\Omega_{b c}^{0}, \Omega_{b c}^{\prime 0}\right)$ the full account of the state mixing effect is necessary.

\section{Results and discussion}

Now we are in a position to give the c.m.m. corrected bag model predictions for magnetic moments of ground state heavy baryons. The results are presented in Tables 3-7. We also compare our predictions with results obtained using various other approaches. These are:

- Simple nonrelativistic quark model (Nonrel) with state mixing in the case of baryons containing three differently flavoured quarks. Predictions for mixed magnetic moments are taken 
from Ref. [3], all other ones were calculated using input values given in [3] $\left(\mu_{u}=-2 \mu_{d}, \mu_{d}=-0.93 \mu_{N^{\prime}}\right.$ $\mu_{s}=-0.61 \mu_{N}, \mu_{c}=0.39 \mu_{N}$, and $\left.\mu_{b}=-0.06 \mu_{N}\right)$ and explicit expressions from Tables $8-10$. Here and further $\mu_{N}$ denotes the nuclear magneton.

- Phenomenological relativistic quark model [15]. The authors of this work studied three forms of relativistic kinematics. For comparison we have picked out the "instant" form.

- Quark model based on the Dirac equation with a confining power-law potential [16].

- Relativistic three-quark model [17].

- Full nonrelativistic calculation using Faddeev formalism with AL1 potential [18].
- Nonrelativistic variational ansatz with the same AL1 potential [19]. For $J=\frac{3}{2}$ baryons (Tables 5 and 7) we group the predictions of Ref. [18] for magnetic moments of $\Omega_{c c c}^{++}$and $\Omega_{b b b}^{-}$with the results obtained in [19] for other baryons in one column. - Nonrelativistic model with screening and effective quark mass [20, 21]. By the way, in this approach the screening effect spoils the quark model relation (24).

- Nonrelativistic hypercentral model [22, 23, Their states $\Xi_{Q}$ are evidently symmetric and should be renamed as $\Xi_{Q}^{\prime}$.

- Chiral constituent quark model [24]. Their prediction for the magnetic moment of a triply

Table 3. Magnetic moments (in nuclear magnetons) of $J=\frac{1}{2}$ charmed baryons calculated in the bag model (Bag) and in other approaches as described in the text.

\begin{tabular}{lcccccccccc}
\hline \multicolumn{1}{c}{ Baryons } & Bag & Nonrel & {$[15]$} & {$[16]$} & {$[17]$} & {$[20]$} & {$[24]$} & {$[25]$} \\
\hline$\Lambda_{c}^{+}$ & 0.411 & 0.39 & 0.40 & 0.341 & 0.42 & 0.37 & 0.39 & 0.37 \\
\hline$\Sigma_{c}^{0}$ & -1.043 & -1.37 & -1.38 & -1.391 & -1.04 & -1.17 & -1.60 & - \\
\hline$\Sigma_{c}^{+}$ & 0.318 & 0.49 & 0.49 & 0.525 & 0.36 & 0.63 & 0.30 & - \\
\hline$\Sigma_{c}^{++}$ & 1.679 & 2.35 & 2.36 & 2.44 & 1.76 & 2.18 & 2.20 & - \\
\hline$\Xi_{c}^{0}$ & 0.421 & 0.41 & 0.41 & 0.341 & 0.39 & 0.36 & 0.28 & 0.32 \\
\hline$\Xi_{c}^{\prime 0}$ & -0.914 & -1.18 & -1.12 & -1.12 & -0.95 & -0.93 & -1.32 & - \\
\hline$\Xi_{c}^{+}$ & 0.257 & 0.20 & 0.40 & 0.341 & 0.41 & 0.37 & 0.40 & 0.42 \\
\hline$\Xi_{c}^{\prime+}$ & 0.591 & 0.89 & 0.75 & 0.796 & 0.47 & 0.76 & 0.76 & - \\
\hline$\Omega_{c}^{0}$ & -0.774 & -0.94 & -0.86 & -0.850 & -0.85 & -0.92 & -0.90 & - \\
\hline$\Xi_{c c}^{+}$ & 0.722 & 0.83 & 0.86 & 0.774 & 0.72 & 0.77 & 0.84 & - \\
\hline$\Xi_{c c}^{++}$ & 0.114 & -0.10 & -0.10 & -0.184 & 0.13 & -0.11 & 0.006 & - \\
\hline$\Omega_{c c}^{+}$ & 0.668 & 0.72 & 0.72 & 0.639 & 0.67 & 0.70 & 0.70 & - \\
\hline$\left|\Sigma_{c}^{+} \rightarrow \Lambda_{c}^{+}\right|$ & 1.182 & 1.61 & - & - & - & 1.54 & 1.56 & - \\
\hline$\left|\Xi_{c}^{\prime 0} \rightarrow \Xi_{c}^{0}\right|$ & 0.013 & 0.08 & - & - & - & 0.13 & 0.31 & - \\
\hline$\left|\Xi_{c}^{\prime+} \rightarrow \Xi_{c}^{+}\right|$ & 1.043 & 1.40 & - & - & - & 1.39 & 1.30 & - \\
\hline
\end{tabular}

Table 4. Magnetic moments (in nuclear magnetons) of $J=\frac{1}{2}$ charmed baryons - continuation of Table 3.

\begin{tabular}{l|c|c|c|c|c|c}
\hline Baryons & Bag & {$[18]$} & {$[19]$} & {$[22]$} & {$[26]$} & {$[28]$} \\
\hline$\Lambda_{c}^{+}$ & 0.411 & 0.341 & - & 0.385 & $0.15 \pm 0.05$ & $0.40 \pm 0.05$ \\
\hline$\Sigma_{c}^{0}$ & -1.043 & -1.435 & - & -1.015 & $-1.6 \pm 0.2$ & - \\
\hline$\Sigma_{c}^{+}$ & 0.318 & 0.548 & - & 0.501 & $0.6 \pm 0.1$ & - \\
\hline$\Sigma_{c}^{++}$ & 1.679 & 2.532 & - & 2.279 & $2.1 \pm 0.3$ & - \\
\hline$\Xi_{c}^{0}$ & 0.421 & 0.360 & - & - & - & $0.35 \pm 0.05$ \\
\hline$\Xi_{c}^{\prime 0}$ & -0.914 & - & - & -0.966 & - & - \\
\hline$\Xi_{c}^{+}$ & 0.257 & 0.211 & - & - & - & $0.50 \pm 0.05$ \\
\hline$\Xi_{c}^{\prime+}$ & 0.591 & - & - & 0.711 & - & - \\
\hline$\Omega_{c}^{0}$ & -0.774 & -0.835 & - & -0.960 & - & - \\
\hline$\Xi_{c c}^{+}$ & 0.722 & 0.784 & 0.785 & 0.860 & - & - \\
\hline$\Xi_{c c}^{++}$ & 0.114 & -0.206 & -0.208 & -0.137 & - & - \\
\hline$\Omega_{c c}^{+}$ & 0.668 & 0.635 & 0.635 & 0.785 & - & - \\
\hline
\end{tabular}


Table 5. Magnetic moments (in nuclear magnetons) of $J=\frac{3}{2}$ charmed baryons calculated in the bag model (Bag) and in other approaches as described in the text.

\begin{tabular}{lccccccccc}
\hline Baryons & Bag & Nonrel & {$[18,19]$} & {$[21]$} & {$[22,23]$} & {$[24]^{*}$} & {$[29]$} \\
\hline$\Sigma_{c}^{*_{0}}$ & -0.958 & -1.47 & - & -1.18 & -0.850 & -1.99 & $-0.81 \pm 0.20$ \\
\hline$\Sigma_{c}^{*+}$ & 1.085 & 1.32 & - & 1.18 & 1.256 & 0.97 & $2.00 \pm 0.46$ \\
\hline$\Sigma_{c}^{*++}$ & 3.127 & 4.11 & - & 3.63 & 3.844 & 3.92 & $4.81 \pm 1.22$ \\
\hline$\Sigma_{c}^{* 0}$ & -0.746 & -1.15 & - & -1.02 & -0.690 & -1.49 & $-0.68 \pm 0.18$ \\
\hline$\Xi_{c}^{*+}$ & 1.270 & 1.64 & - & 1.39 & 1.517 & 1.59 & $1.68 \pm 0.42$ \\
\hline$\Omega_{c}^{* 0}$ & -0.547 & -0.83 & - & -0.84 & -0.867 & -0.86 & $-0.62 \pm 0.18$ \\
\hline$\Xi_{c c}^{*+}$ & 0.163 & -0.15 & -0.311 & 0.035 & -0.168 & -0.47 & - \\
\hline$\Xi_{c}^{*++}$ & 2.001 & 2.64 & 2.670 & 2.52 & 2.755 & 2.66 & - \\
\hline$\Omega_{c c}^{*+}$ & 0.332 & 0.17 & 0.139 & 0.21 & 0.121 & 0.14 & - \\
\hline$\Omega_{c c c}^{++}$ & 1.138 & 1.17 & 1.023 & 1.16 & 1.189 & 1.17 & - \\
\hline
\end{tabular}

${ }^{*}$ Value for $\Omega_{c c c}^{++}$corrected as deduced and explained in the text.

Table 6. Magnetic moments (in nuclear magnetons) of $J=\frac{1}{2}$ bottom baryons calculated in the bag model (Bag) and in other approaches as described in the text.

\begin{tabular}{|c|c|c|c|c|c|c|c|c|}
\hline Baryons & Bag & Nonrel & {$[16]$} & {$[17]^{* *}$} & {$[18]$} & {$[19]$} & {$[22,23]$} & {$[27,28]$} \\
\hline$\Lambda_{\mathrm{b}}^{0}$ & -0.066 & -0.06 & - & -0.06 & -0.060 & - & -0.064 & $-0.18 \pm 0.05$ \\
\hline$\Sigma_{\mathrm{b}}^{-}$ & -0.778 & -1.22 & -1.256 & -1.01 & -1.305 & - & -1.047 & - \\
\hline$\Sigma_{\mathrm{b}}^{0}$ & 0.422 & 0.64 & 0.659 & 0.53 & 0.682 & - & 0.592 & - \\
\hline$\Sigma_{b}^{+}$ & 1.622 & 2.50 & 2.575 & 2.07 & 2.669 & - & 2.229 & - \\
\hline$\Xi_{\mathrm{b}}^{-}$ & -0.063 & -0.05 & - & -0.06 & -0.055 & - & - & $-0.08 \pm 0.02$ \\
\hline$\Xi_{b}^{\prime-}$ & -0.660 & -1.02 & -0.985 & -0.91 & - & - & -0.902 & - \\
\hline$\Xi_{b}^{0}$ & -0.100 & -0.11 & - & -0.06 & -0.086 & - & - & $-0.045 \pm 0.005$ \\
\hline$\Xi_{\mathrm{b}}^{\prime 0}$ & 0.556 & 0.90 & 0.930 & 0.66 & - & - & 0.766 & - \\
\hline$\Omega_{\mathrm{b}}^{-}$ & -0.545 & -0.79 & -0.714 & -0.82 & -0.703 & - & -0.960 & - \\
\hline$\Xi_{\mathrm{bc}}^{0}$ & 0.068 & 0.13 & - & 0.42 & 0.058 & 0.518 & 0.477 & - \\
\hline$\Xi_{\mathrm{bc}}^{\prime 0}$ & -0.236 & -0.53 & -0.390 & -0.76 & - & -0.993 & - & - \\
\hline$\Xi_{b c}^{+}$ & -0.157 & -0.25 & - & -0.12 & -0.198 & -0.475 & -0.400 & - \\
\hline$\Xi_{\mathrm{bc}}^{\prime+}$ & 1.093 & 1.71 & 1.525 & 1.52 & - & 1.990 & - & - \\
\hline$\Omega_{\mathrm{bc}}^{0}$ & 0.034 & 0.08 & -0.119 & 0.45 & 0.009 & 0.368 & 0.397 & - \\
\hline$\Omega_{\mathrm{bc}}^{\prime 0}$ & -0.106 & -0.27 & - & -0.61 & - & -0.542 & - & - \\
\hline$\Omega_{\mathrm{bcc}}^{+}$ & 0.505 & 0.54 & 0.476 & 0.53 & 0.475 & - & 0.502 & - \\
\hline$\Xi_{\mathrm{bb}}^{-}$ & 0.086 & 0.23 & 0.236 & 0.18 & 0.251 & 0.251 & 0.190 & - \\
\hline$\Xi_{\mathrm{bb}}^{0}$ & -0.432 & -0.70 & -0.722 & -0.53 & -0.742 & -0.742 & -0.657 & - \\
\hline$\Omega_{\mathrm{bb}}^{-}$ & 0.043 & 0.12 & 0.100 & 0.04 & 0.101 & 0.101 & 0.109 & - \\
\hline$\Omega_{\mathrm{bbc}}^{0}$ & -0.205 & -0.21 & -0.197 & -0.20 & -0.193 & - & -0.203 & - \\
\hline$\left|\Sigma_{\mathrm{b}}^{0} \rightarrow \Lambda_{\mathrm{b}}^{0}\right|$ & 1.052 & 1.61 & - & - & - & - & - & - \\
\hline$\left|\Xi_{b}^{\prime} \rightarrow \Xi_{b}^{-}\right|$ & 0.082 & 0.16 & - & - & - & - & - & - \\
\hline$\left|\Xi_{\mathrm{b}}^{\prime 0} \rightarrow \Xi_{\mathrm{b}}^{0}\right|$ & 0.917 & 1.41 & - & - & - & - & - & - \\
\hline$\left|\Xi_{\mathrm{bc}}^{\prime 0} \rightarrow \Xi_{\mathrm{bc}}^{0}\right|$ & 0.508 & 0.70 & - & - & - & - & - & - \\
\hline$\left|\Xi_{\mathrm{bc}}^{\prime+} \rightarrow \Xi_{\mathrm{bc}}^{+}\right|$ & 0.277 & 0.62 & - & - & - & - & - & - \\
\hline$\left|\Omega_{\mathrm{bc}}^{\prime 0} \rightarrow \Omega_{\mathrm{bc}}^{0}\right|$ & 0.443 & 0.56 & - & - & - & - & - & - \\
\hline
\end{tabular}

${ }^{* *}$ Primes on states of $\Xi_{b c}$ and $\Omega_{b c}$ different from [17] (opposite), as explained in the text. 
heavy baryon $\Omega_{c c c}^{++}$is an order of magnitude lower than predictions obtained in all other Refs. We guess that authors of [24] have used for valence contribution the value $0.165 \mu_{N}$ instead of $1.165 \mu_{N}$. In Table 5 we have changed their value for magnetic moment of $\Omega_{c c c}^{++}$from $0.155 \mu_{N}$ to $1.17 \mu_{N}$ on our own responsibility.

- Chiral perturbation theory [25].

- QCD spectral sum rules [26].

- Light cone QCD sum rules [27-29].

Care must be taken when one tries to compare various expressions and results of the calculations with earlier works because of some mess-up in the notations of primed and unprimed states for the single heavy baryons $\Xi_{Q}$ and $\Xi_{Q}^{\prime}$ Usually [1] the physical $\Xi_{Q}$ state is assumed to be that which contains a pair of light quarks mostly in $S=0$ (antisymmetric) state $\left|\Xi_{Q}\right\rangle \sim\left|\left[q_{1} q_{2}\right] Q\right\rangle$ where $q_{i}$ denotes the light and $Q$ the heavy quarks. Respectively, the primed state $\Xi_{Q}^{\prime}$ is mostly $S=1$ (symmetric) state $\left|\Xi_{Q}^{\prime}\right\rangle \sim\left|\left\{q_{1} q_{2}\right\} Q\right\rangle$. Often the notations $\Xi_{Q}\left(\Xi_{Q}^{\prime}\right)$ are simply used to denote pure antisymmetric (symmetric) states. When the quark model was young the opposite convention was in use. Such old-fashioned (opposite) convention has been used for designating the primed states in Refs. [3, 9, 16. This circumstance must be taken into account when comparing their results with ours.

Some complications arise when we want to compare our predictions for doubly heavy bary- ons $\Xi_{b c} \Xi_{b c}^{\prime}$ and $\Omega_{b c}, \Omega_{b c}^{\prime}$ with the unmixed results obtained using the quark ordering scheme representing heavy diquark picture [17, 19, 22, 30], in which the spins of the two heaviest quarks are coupled to form a symmetric $\left\{Q_{1} Q_{2}\right\}$ or antisymmetric $\left[Q_{1} Q_{2}\right]$ diquark. At first sight such scheme seems to follow the recipe of Ref. [3] that the two quarks closest in mass must be (anti)symmetrized as the first two. But the fact that the two quarks are the heaviest does not mean that they are the closest in mass. With respect to the colour-hyperfine interaction $u$ (or $d$ ) and $c$ quarks are closer than $c$ and $b$ (see Table 2). Meanwhile, in the case of two identical heavy quarks the heavy diquark picture is a perfect choice. Of course, we can compare their predictions with our unmixed results corresponding to the quark ordering $\left(Q_{1} Q_{2}\right) q$ and obtain good qualitative agreement. However, our unmixed states are not physical states; therefore, such comparison between presumably physical and nonphysical states seems to be unsatisfactory. On the other hand, we see from Table 2 that our unprimed state is predominantly the symmetric heavy diquark state with some (not very small) admixture of an antisymmetric state, i.e. $|B\rangle=C_{1}\left|\left[Q_{1} Q_{2}\right] q_{3}\right\rangle$ $+C_{2}\left|\left\{Q_{1} Q_{2}\right\} q_{3}\right\rangle$, where $C_{2}^{2}>C_{1}^{2}$. So, it makes some sense to denote a symmetric heavy diquark state as $|B\rangle$ and an antisymmetric one as $\left|B^{\prime}\right\rangle$. Such convention has been chosen in Refs. [19, 22, 30]. However,

Table 7. Magnetic moments (in nuclear magnetons) of $J=\frac{3}{2}$ bottom baryons calculated in the bag model (Bag) and in other approaches as described in the text.

\begin{tabular}{lcccccc}
\hline \multicolumn{1}{c}{ Baryons } & Bag & Nonrel & {$[18,19]$} & {$[22,23]$} & {$[29]$} \\
\hline$\sum_{b}^{*-}$ & -1.271 & -1.92 & - & -1.657 & $-1.50 \pm 0.36$ \\
\hline$\sum_{b}^{* 0}$ & 0.537 & 0.87 & - & 0.792 & $0.50 \pm 0.15$ \\
\hline$\sum_{b}^{*+}$ & 2.346 & 3.56 & - & 3.239 & $2.52 \pm 0.50$ \\
\hline$\Xi_{b}^{*-}$ & -1.088 & -1.60 & - & -1.098 & $-1.42 \pm 0.35$ \\
\hline$\Xi_{b}^{* 0}$ & 0.690 & 1.19 & - & 1.042 & $0.50 \pm 0.15$ \\
\hline$\Omega_{b-}^{*-}$ & -0.919 & -1.28 & - & -1.201 & $-1.40 \pm 0.35$ \\
\hline$\Xi_{b c}^{* 0}$ & -0.257 & -0.60 & -0.712 & -0.568 & - \\
\hline$\Xi_{b c}^{*+}$ & 1.414 & 2.19 & 2.270 & 2.052 & - \\
\hline$\Omega_{b c}^{* 0}$ & -0.111 & -0.28 & -0.261 & -0.317 & - \\
\hline$\Omega_{b c c}^{*+}$ & 0.659 & 0.72 & - & 0.651 & - \\
\hline$\Xi_{b \bar{b}}^{*-}$ & -0.652 & -1.05 & -1.110 & -0.952 & - \\
\hline$\Xi_{b b}^{* 0}$ & 0.916 & 1.74 & 1.870 & 1.577 & - \\
\hline$\Omega_{b \bar{b}}^{*-19}$ & -0.73 & -0.662 & 0.711 & - \\
\hline$\Omega_{b b c}^{* 0}$ & -0.522 & 0.27 & - & 0.216 & - \\
\hline$\Omega_{b b b}^{-}$ & 0.225 & -0.18 & -0.180 & -0.195 & - \\
\hline
\end{tabular}


in Ref. [17] the opposite convention has been used. For convenience and in order to have a more consistent representation we have renamed (in Table 6) their $\Xi_{b c}$ and $\Omega_{b c}$ states as $\Xi_{b c}^{\prime}$ and $\Omega_{b c}^{\prime}$ (and correspondingly $\Xi_{b c}^{\prime}, \Omega_{b c}^{\prime}$ as $\left.\Xi_{b c}, \Omega_{b c}\right)$.

Now let us focus on the results presented in Tables 3-7. The first impression is that almost all collected predictions (with only several exceptions) as a whole give us a relatively consistent picture. This is the consequence of underlying symmetry shared by the models. But can we understand the differences? Not always, but sometimes we can. As a first step it is not a bad idea to compare our bag model predictions with the results given by a simple nonrelativistic model (columns 3 of Tables 3, 5-7). We see that the qualitative picture in both variants is similar, while numerical values differ, sometimes substantially. Maybe the most intriguing feature is the opposite sign of predicted magnetic moments in the bag and nonrelativistic models for $\Xi_{c c}^{++}$and $\Xi_{c c}^{*+}$ baryons. The inspection of predictions presented in Tables 3-5 shows that there is no common agreement on the signs of these magnetic moments. The explanation of such disagreement is quite simple. The magnetic moment of $\Xi_{c c}^{++}$is given by the expression $\mu\left(\Xi_{c c}^{++}\right)=\frac{2}{9}\left(4 \bar{\mu}_{c}-\bar{\mu}_{u}\right)$ (see Table 8$)$. The sign of this magnetic moment depends on what is "stronger" - two $c$ quarks or one $u$ quark. In the nonrelativistic model the $u$ quark overcomes the $c$-duet, while in our bag model the $c$ quarks defeat a single $u$ quark. The magnetic moment of $\Xi_{c c}^{*+}$ is related to $\mu\left(\Xi_{c c}^{++}\right)$by Eq. (24), therefore its sign must be the same.

Contrary to the naive nonrelativistic model, in the bag model the magnetic moment of a quark depends on a baryon, the owner of this quark. There are two effects that make the quark magnetic moments smaller in the case of heavy baryons. Firstly, the dependence of the quark magnetic moment on the bag radius. A heavier baryon has a smaller bag radius and this leads to a smaller quark magnetic moment in accordance. The second reason is the relative strength of the c.m.m. corrections. In the case of light hadrons these corrections are stronger and therefore lead to larger magnetic moments than in the case of heavy baryons. For example, in the proton the value of the c.m.m. corrected magnetic moment of $u$ quark is $\mu_{u}(P)=-1.924 \mu_{N}$, while in the $\Xi_{b}$ baryon it is only $\mu_{u}\left(\Xi_{b}\right)=1.168 \mu_{N}$. For heavier baryon $\Xi_{b b}$ it becomes even smaller, $\mu_{u}\left(\Xi_{b b}\right)=1.036 \mu_{N}$. The similar feature of the mag- netic moment of the strange quark also cannot be ignored. For example, $\mu_{s}\left(\Omega_{b}\right)=-0.425 \mu_{N}$, while $\mu_{s}\left(\Omega_{b b}\right)=-0.390 \mu_{N}$. It is this difference that was at the root of the failure of the quark model relation $3\left[4 \mu\left(\Omega_{b b}^{-}\right)+\mu\left(\Omega_{b}^{-}\right)\right]=5\left[2 \mu\left(\Omega_{b b}^{*-}\right)-\mu\left(\Omega_{b}^{*-}\right)\right]$ in Sec. 3. For charmed quarks the dependence diminishes, but remains appreciable. For example, $\mu_{c}\left(\Lambda_{c}^{+}\right)=0.411 \mu_{N}$, while in the triply heavy baryon $\mu_{b}\left(\Omega_{c c c}^{++}\right)=0.379 \mu_{N}$. In contrast, the magnetic moments of bottom quarks are almost insensible to the baryon they live in $\left(\mu_{b}\left(\Lambda_{b}^{0}\right)=-0.066 \mu_{N}\right.$ and $\mu_{b}\left(\Omega_{b b b}^{-}\right)=-0.065 \mu_{N}$, for example). There are several baryons (e. g., $\Lambda_{c}, \Omega_{c c c}, \Lambda_{b}, \Omega_{b c c}, \Omega_{b c c}^{*}, \Omega_{b b c}, \Omega_{b b c}^{*}, \Omega_{b b b}$ ) the magnetic moments of which depend on the magnetic moments of the heavy quarks only. We expect that in these cases the results obtained in the bag model and in the nonrelativistic one must be similar. The differences indeed do not exceed $10 \%$. In all other cases the bag model predicted values of magnetic moments are smaller than corresponding nonrelativistic results (sometimes distinctly).

The magnetic moments of heavy baryons are unlikely to be measured in the nearest future. In such situation any indirect estimate of these quantities could be helpful. Some useful information can be extracted from the mass spectra of baryons. Magnetic moments of quarks are proportional to the chromomagnetic moments which determine the colour-hyperfine splitting of baryon masses. Using this fact the magnetic moments of $\Lambda_{c}$ and $\Lambda_{b}$ can be obtained [31]. The predictions are $\mu_{c}\left(\Lambda_{c}^{+}\right)=0.43 \mu_{N}$ and $\mu_{b}\left(\Lambda_{b}^{0}\right)=-0.067 \mu_{N}$, in excellent coincidence with our results. These values are also consistent with almost all other predictions with the exception of spectral sum rules [26], where the source of deviation is the contribution from higher-dimension condensates.

We think it could be reasonable to continue the comparison of bag model predictions with the results obtained in other approaches with the simplest case of $J=\frac{3}{2}$ bottom baryons (Table 7). We see immediately that our predictions are compatible with the light cone sum rules [29], while the agreement between nonrelativistic model predictions and light cone sum rules is not so good. The results obtained using a hypercentral model [22, 23] are, as a rule, somewhere between naive nonrelativistic predictions and ours. For example, their predictions for the magnetic moments of triply heavy baryons agree well with our results, while in other 
cases they are closer to the predictions of simple nonrelativistic model. Variational calculations [19] do not differ substantially from the results obtained using a naive nonrelativistic model.

The situation is similar for the $J=\frac{3}{2}$ charmed baryons (Table 5). Bag model predictions for $\Sigma_{c}^{* 0}$, $\Xi_{c}^{*_{0}}, \Xi_{c}^{*+}$, and $\Omega_{c}^{*_{0}}$ are compatible with the light cone sum rules again, while in the case of $\Sigma_{c}^{*+}$ and $\Sigma_{c}^{*++}$ our values are somewhat lower than the light cone results. The hypercentral predictions on the average are closer to naive nonrelativistic results, but these for $\Sigma_{c}^{*_{0}}, \Xi_{c}^{*_{0}}$ are closer to ours. The chiral model [24] predicts larger values in all cases, even larger than nonrelativistic results. Variational results are close to naive nonrelativistic predictions in this case again.

Before proceeding with the case of $J=\frac{1}{2}$ baryons let us revert for a moment to the comparison of our bag model predictions with the predictions obtained in the simple nonrelativistic approach. There exists one more correspondence between bag model results and the nonrelativistic predictions. In both cases for the baryons containing three differently flavoured quarks the state mixing effect caused by the colour-hyperfine interaction was taken into account. This is a significant improvement which can lead to substantial shifts of the predicted magnetic moments. For the mixed magnetic moments we have a reasonable qualitative agreement between predictions obtained in both models. Another approach in which the state mixing is taken into account (by definition) is the Faddeev formalism [18]. The predictions obtained using this method do not differ very much from naive nonrelativistic results. To see what is the importance of the state mixing effect one can compare the result of Ref. [18] for baryons $\Xi_{b c}^{0}, \Xi_{b c}^{+}$, and $\Omega_{b c}^{0}$ with the corresponding results obtained in Ref. [19] where this mixing was ignored. Note that predicted magnetic moments can differ by almost 40 times (in extreme case of $\Omega_{b c}^{0}$. The importance of this effect for the models with effective colour-hyperfine interaction has been known for years [3]; nevertheless, in many calculations it was systematically ignored for various reasons (peculiarities of the model, technical difficulties, etc.) This seems to be the case for all other calculations we are going to compare our predictions with. A special exception is pure chiral models in which the state mixing of this type is naturally absent because of a different type of effective interaction used. This is the reason of some quali- tative difference between predictions for magnetic moments of heavy baryons obtained in chiral approach and in models based on the effective colourhyperfine interaction. Therefore, when we compare our results for baryons $\Xi_{c}^{+}, \Xi_{c}^{\prime+} ; \Xi_{b}^{0}, \Xi_{b}^{\prime 0} ; \Xi_{b c}^{0}, \Xi_{b c}^{\prime 0} ; \Xi_{b c}^{+}$, $\Xi_{b c}^{\prime+}$; and $\Omega_{b c}^{0}, \Omega_{b c}^{\prime 0}$ with predictions of others we must keep all theses circumstances in mind.

With these not very short preliminaries we can now proceed comparing our predictions with other results. We see that all models, as expected, give very similar predictions in the case of triply heavy $J=\frac{1}{2}$ baryons $\Omega_{b c c}$ and $\Omega_{b b c}$. The agreement of bag model results with the sum rules in the case of $J=\frac{1}{2}$ baryons is not so good. For example, for $\Xi_{c}^{0}$ and $\Xi_{c}^{+}$baryons the light cone sum rules predict the values similar to results obtained in the chiral model [24] and in a chiral perturbation theory [25]. Our predictions in these cases differ substantially. The state mixing effect acts in opposite direction as chiral corrections. Could these two effects if applied simultaneously compensate each other? In any case it should depend on the model. Possibly it could happen in models with some mixture of one-gluonexchange and Goldstone-boson-exchange induced interactions. Hypercentral predictions in the charm sector are again somewhere between simple nonrelativistic results and ours (some closer to ours, some to naive nonrelativistic), but for the bottom baryons they are closer to the predictions obtained in a simple nonrelativistic approach. Almost all other predictions are closer to nonrelativistic results and provide larger values than ours. For example, the predictions of Ref. [15] for unmixed moments are almost indistinguishable from the naive nonrelativistic predictions. However, we found out, with some surprise, that our predictions for magnetic moments of $J=\frac{1}{2}$ baryons (at least unmixed) resemble the results obtained in Ref. [17] including a positive sign of $\mu\left(\Xi_{c c}^{++}\right)$. The models are very different, and we have no reasonable explanation of this resemblance. Could the reason be a common relativistic nature? Initially, both of them were formulated as relativistic nonlocal field theories. It may be, but the other two relativistic models [15, 16, behave very much like their nonrelativistic neighbours.

In summary, we have used the improved bag model to calculate magnetic moments of $J=\frac{1}{2}$ and $J=\frac{3}{2}$ heavy baryons without introducing any new parameters and obtained encouraging results. The status of quark model relations connecting 
magnetic moments of various baryons was revisited. A part of work was devoted to study the state mixing induced by the colour-hyperfine interaction. It has been shown that for the baryons $\Xi_{c}^{+}, \Xi_{c}^{\prime+}$; $\Xi_{b}^{0}, \Xi_{b}^{\prime 0} ; \Xi_{b c}^{0}, \Xi_{b c}^{\prime 0}, \Xi_{b c}^{+}, \Xi_{b c}^{\prime+}$ and $\Omega_{b c}^{0}, \Omega_{b c}^{\prime 0}$ this mixing leads to appreciable shifts of the unmixed magnetic moments and, in order to have a consistent description, must be taken into account.

\section{Appendix. Explicit expressions for the magnetic moments of heavy baryons}

In the three tables below we present expressions for the magnetic moments of spin $\frac{1}{2}$ and spin $\frac{3}{2}$ charmed and bottom baryons. For simplicity the shorthand notations $\left(\mu_{q} \rightarrow q, \bar{\mu}_{q} \rightarrow \bar{q}\right)$ are used. The entries in columns 4 were obtained by setting $(u, c)=\frac{2}{3}(\bar{u}, \bar{c}), \quad(d, s, b)=-\frac{1}{3}(\bar{d}, \bar{s}, \bar{b})$, and assuming isospin symmetry $(\bar{u}=\bar{d})$.

In the case of baryons containing three quarks of different flavours the quark arrangement with

Table 8. Composition of $J=\frac{1}{2}$ charmed baryon magnetic moments in terms of magnetic moments of individual quarks (column 3) and corresponding reduced quantities (column 4).

\begin{tabular}{lcccc}
\hline Particles & quark ordering & $\mu_{\mathrm{B}}^{0}$ & $\mu_{\mathrm{B}}^{0}$ \\
\hline$\Lambda_{c}^{+}$ & {$[u d] c$} & $c$ & $\frac{2}{3} \bar{c}$ \\
\hline$\Sigma_{c}^{+}$ & $\{u d\} c$ & $\frac{1}{3}(2 u+2 d-c)$ & $\frac{2}{9}(\bar{u}-\bar{c})$ \\
\hline$\Sigma_{c}^{+} \rightarrow \Lambda_{c}^{+}$ & $\begin{array}{c}\{u d\} c \rightarrow \\
{[u d] c}\end{array}$ & $\frac{1}{\sqrt{3}}(d-u)$ & $-\frac{1}{\sqrt{3}} \bar{u}$ \\
\hline$\Sigma_{c}^{0}$ & $d d c$ & $\frac{1}{3}(4 d-c)$ & $-\frac{2}{9}(2 \bar{u}+\bar{c})$ \\
\hline$\Sigma_{c}^{++}$ & $u u c$ & $\frac{1}{3}(4 u-c)$ & $\frac{2}{9}(4 \bar{u}-\bar{c})$ \\
\hline$\Xi_{c}^{0} \Xi_{c}^{\prime 0}$ & {$[d s] c$} & $c$ & $\frac{2}{3} \bar{c}$ \\
\hline$\prime$ & $\{d s\} c$ & $\frac{1}{3}(2 d+2 s-c)$ & $-\frac{2}{9}(\bar{u}+\bar{s}+\bar{c})$ \\
\hline$\prime \prime$ & $\{d s\} c \rightarrow$ & $\frac{1}{\sqrt{3}}(s-d)$ & $\frac{1}{3 \sqrt{3}}(\bar{u}-\bar{s})$ \\
\hline$\Xi_{c}^{+}, \Xi_{c}^{\prime+}$ & {$[d s] c$} & $c$ & $\frac{2}{3} \bar{c}$ \\
\hline$\prime \prime$ & $\{u s] c$ & \multicolumn{1}{c}{$c$} & $\frac{1}{3}(2 u+2 s-c)$ & $\frac{2}{9}(2 \bar{u}-\bar{s}-\bar{c})$ \\
\hline$\prime \prime$ & $\{u s\} c \rightarrow$ & $\frac{1}{\sqrt{3}}(s-u)$ & $-\frac{1}{3 \sqrt{3}}(2 \bar{u}+\bar{s})$ \\
\hline$\Omega_{c}^{0}$ & $s u s] c$ & $\frac{1}{3}(4 s-c)$ & $-\frac{2}{9}(2 \bar{s}+\bar{c})$ \\
\hline$\Xi_{c c}^{+}$ & $c c d$ & $\frac{1}{3}(4 c-d)$ & $\frac{1}{9}(\bar{u}+8 \bar{c})$ \\
\hline$\Xi_{c c}^{++}$ & $c c u$ & $\frac{1}{3}(4 c-u)$ & $-\frac{2}{9}(\bar{u}-4 \bar{c})$ \\
\hline$\Omega_{c c}^{+}$ & $c c s$ & $\frac{1}{3}(4 c-s)$ & $\frac{1}{9}(\bar{s}+8 \bar{c})$ \\
\hline & & &
\end{tabular}

the heaviest quark placed as the third one in the spin coupling scheme has been chosen. The entries of the column 3 for other arrangements can be easily obtained by simple quark renaming.

Table 9. Composition of $J=\frac{1}{2}$ bottom baryon magnetic moments in terms of magnetic moments of individual quarks (column 3) and corresponding reduced quantities (column 4).

\begin{tabular}{l|c|c|c}
\hline Particles & $\begin{array}{c}\text { quark } \\
\text { ordering }\end{array}$ & $\mu_{\mathrm{B}}^{0}$ & $\mu_{\mathrm{B}}^{0}$ \\
\hline$\Lambda_{b}^{0}$ & {$[u d] b$} & $b$ & $-\frac{1}{3} \bar{b}$ \\
\hline$\Sigma_{b}^{0}$ & $\{u d\} b$ & $\frac{1}{3}(2 u+2 d-b)$ & $\frac{1}{9}(2 \bar{u}+\bar{b})$ \\
\hline$\Sigma_{b}^{0} \rightarrow \Lambda_{b}^{0}$ & $\begin{array}{c}\{u d\} b \rightarrow \\
{[u d] b}\end{array}$ & $\frac{1}{\sqrt{3}}(d-u)$ & $-\frac{1}{\sqrt{3}} \bar{u}$ \\
\hline$\Sigma_{b}^{-}$ & $d d b$ & $\frac{1}{3}(4 d-b)$ & $-\frac{1}{9}(4 \bar{u}-\bar{b})$ \\
\hline$\Sigma_{b}^{+}$ & $u u b$ & $\frac{1}{3}(4 u-b)$ & $\frac{1}{9}(8 \bar{u}+\bar{b})$ \\
\hline$\Xi_{b}^{-}, \Xi_{b}^{\prime}$ & {$[d s] b$} & $b$ & $-\frac{1}{3} \bar{b}$ \\
\hline$\prime \prime$ & $\{d s\} b$ & $\frac{1}{3}(2 d+2 s-b)$ & $-\frac{1}{9}(2 \bar{u}+2 \bar{s}-\bar{b})$ \\
\hline$\prime \prime$ & $\{d s\} b \rightarrow$ & $\frac{1}{\sqrt{3}}(s-d)$ & $\frac{1}{3 \sqrt{3}}(\bar{u}-\bar{s})$ \\
\hline
\end{tabular}

\begin{tabular}{lccc}
\hline$\Xi_{b}^{0}, \Xi_{b}^{\prime 0}$ & {$[u s] b$} & $b$ & $-\frac{1}{3} \bar{b}$ \\
\hline$\prime$ & $\{u s\} b$ & $\frac{1}{3}(2 u+2 s-b)$ & $\frac{1}{9}(4 \bar{u}-2 \bar{s}+\bar{b})$ \\
\hline$\prime \prime$ & $\begin{array}{c}\{u s\} b \rightarrow \\
{[u s] b}\end{array}$ & $\frac{1}{\sqrt{3}}(s-u)$ & $-\frac{1}{3 \sqrt{3}}(2 \bar{u}+\bar{s})$ \\
\hline$\Omega_{b}^{-}$ & $s s b$ & $\frac{1}{3}(4 s-b)$ & $-\frac{1}{9}(4 \bar{s}-\bar{b})$ \\
\hline$\Xi_{b c}^{0} \Xi_{b c}^{\prime 0}$ & {$[d c] b$} & $b$ & $-\frac{1}{3} \bar{b}$ \\
\hline$\prime$ & $\{d c\} b$ & $\frac{1}{3}(2 d+2 c-b)$ & $-\frac{1}{9}(2 \bar{u}-4 \bar{c}-\bar{b})$ \\
\hline$\prime$ & $\{d c\} b \rightarrow$ & $\frac{1}{\sqrt{3}}(c-d)$ & $\frac{1}{3 \sqrt{3}}(\bar{u}+2 \bar{c})$ \\
\hline
\end{tabular}

\begin{tabular}{lccc}
\hline$\Xi_{b c}^{+} \Xi_{b c}^{\prime+}$ & {$[u c] b$} & $b$ & $-\frac{1}{3} \bar{b}$ \\
\hline$\prime$ & $\{u c\} b$ & $\frac{1}{3}(2 u+2 c-b)$ & $\frac{1}{9}(4 \bar{u}+4 \bar{c}+\bar{b})$ \\
\hline$\prime$ & $\begin{array}{c}\{u c\} b \rightarrow \\
{[u c] b}\end{array}$ & $\frac{1}{\sqrt{3}}(c-u)$ & $\frac{2}{3 \sqrt{3}}(\bar{u}-\bar{c})$ \\
\hline$\Omega_{b c}^{0}, \Omega_{b c}^{\prime 0}$ & {$[s c] b$} & $b$ & $-\frac{1}{3} \bar{b}$ \\
\hline$\prime$ & $\{s c\} b$ & $\frac{1}{3}(2 s+2 c-b)$ & $-\frac{1}{9}(2 \bar{s}-4 \bar{c}-\bar{b})$ \\
\hline$\prime \prime$ & $\begin{array}{c}\{s c\} b \rightarrow \\
{[s c] b}\end{array}$ & $\frac{1}{\sqrt{3}}(c-s)$ & $\frac{1}{3 \sqrt{3}}(\bar{s}+2 \bar{c})$ \\
\hline$\Omega_{b c c}^{+}$ & $c c b$ & $\frac{1}{3}(4 c-b)$ & $\frac{1}{9}(8 \bar{c}-\bar{b})$ \\
\hline$\Xi_{b b}^{-}$ & $b b d$ & $\frac{1}{3}(4 b-d)$ & $\frac{1}{9}(\bar{u}-4 \bar{b})$ \\
\hline$\Xi_{b b}^{0}$ & $b b u$ & $\frac{1}{3}(4 b-u)$ & $-\frac{2}{9}(\bar{u}+2 \bar{b})$ \\
\hline$\Omega_{b b}^{-}$ & $b b s$ & $\frac{1}{3}(4 b-s)$ & $\frac{1}{9}(\bar{s}-4 \bar{b})$ \\
\hline$\Omega_{b b c}^{0}$ & $b b c$ & $\frac{1}{3}(4 b-c)$ & $-\frac{2}{9}(\bar{c}+2 \bar{b})$ \\
\hline & & &
\end{tabular}


Table 10. Composition of $J=\frac{3}{2}$ charmed and bottom baryon magnetic moments in terms of magnetic moments of individual quarks (column 3) and corresponding reduced quantities (column 4).

\begin{tabular}{|c|c|c|c|}
\hline Particles & $\begin{array}{l}\text { quark } \\
\text { content }\end{array}$ & $\mu_{\mathrm{B}}^{0}$ & $\mu_{\mathrm{B}}^{0}$ \\
\hline$\Sigma_{c}^{*_{0}}$ & $d d c$ & $2 d+c$ & $-\frac{2}{3}(\bar{u}-\bar{c})$ \\
\hline$\Sigma_{c}^{*+}$ & $\{u d\} c$ & $u+d+c$ & $\frac{1}{3}(\bar{u}+2 \bar{c})$ \\
\hline$\Sigma_{c}^{*++}$ & $u u c$ & $2 u+c$ & $\frac{2}{3}(2 \bar{u}+\bar{c})$ \\
\hline$\Xi_{c}^{* 0}$ & $\{d s\} c$ & $d+s+c$ & $\frac{1}{3}(2 \bar{c}-\bar{u}-\bar{s})$ \\
\hline$\Xi_{c}^{*+}$ & $\{u s\} c$ & $u+s+c$ & $\frac{1}{3}(2 \bar{c}+2 \bar{u}-\bar{s})$ \\
\hline$\Omega_{c}^{* 0}$ & $s s c$ & $2 s+c$ & $-\frac{2}{3}(\bar{s}-\bar{c})$ \\
\hline$\Xi_{c c}^{*+}$ & $c c d$ & $2 c+d$ & $-\frac{1}{3}(\bar{u}-4 \bar{c})$ \\
\hline$\Xi_{c c}^{*++}$ & $c c u$ & $2 c+u$ & $\frac{2}{3}(\bar{u}+2 \bar{c})$ \\
\hline$\Omega_{c c}^{*+}$ & $c c s$ & $2 c+s$ & $\frac{1}{3}(4 \bar{c}-\bar{s})$ \\
\hline$\Omega_{c c c}^{++}$ & $c c c$ & $3 c$ & $2 \overline{\mathrm{c}}$ \\
\hline$\Sigma_{b}^{*+}$ & $u u b$ & $2 u+b$ & $\frac{1}{3}(4 \bar{u}-\bar{b})$ \\
\hline$\Sigma_{b}^{* 0}$ & $\{u d\} b$ & $u+d+b$ & $\frac{1}{3}(\bar{u}-\bar{b})$ \\
\hline$\Sigma_{b}^{*-}$ & $d d b$ & $2 d+b$ & $-\frac{1}{3}(2 \bar{u}+\bar{b})$ \\
\hline$\Xi_{b}^{* 0}$ & $\{u s\} b$ & $u+s+b$ & $\frac{1}{3}(2 \bar{u}-\bar{s}-\bar{b})$ \\
\hline$\Xi_{b}^{*-}$ & $\{d s\} b$ & $d+s+b$ & $-\frac{1}{3}(\bar{u}+\bar{s}+\bar{b})$ \\
\hline$\Omega_{b}^{*-}$ & $s s b$ & $2 s+b$ & $-\frac{1}{3}(2 \bar{s}+\bar{b})$ \\
\hline$\Xi_{b c}^{*+}$ & $\{u c\} b$ & $u+c+b$ & $\frac{1}{3}(2 \bar{u}+2 \bar{c}-\bar{b})$ \\
\hline$\Xi_{b c}^{* 0}$ & $\{d c\} b$ & $d+c+b$ & $-\frac{1}{3}(\bar{u}+\bar{b}-2 \bar{c})$ \\
\hline$\Omega_{b c}^{\star 0}$ & $\{s c\} b$ & $s+c+b$ & $-\frac{1}{3}(\bar{s}+\bar{b}-2 \bar{c})$ \\
\hline$\Omega_{b c c}^{*+}$ & $c c b$ & $2 c+b$ & $\frac{1}{3}(4 \bar{c}-\bar{b})$ \\
\hline$\Xi_{b b}^{\star 0}$ & $b b u$ & $2 b+u$ & $\frac{2}{3}(\bar{u}-\bar{b})$ \\
\hline$\Xi_{b b}^{*-}$ & $b b d$ & $2 b+d$ & $-\frac{1}{3}(\bar{u}+2 \bar{b})$ \\
\hline$\Omega_{b b}^{*-}$ & $b b s$ & $2 b+s$ & $-\frac{1}{3}(\bar{s}+2 \bar{b})$ \\
\hline$\Omega_{b b c}^{* 0}$ & $b b c$ & $2 b+c$ & $\frac{2}{3}(\bar{c}-\bar{b})$ \\
\hline$\Omega_{b b b}^{-}$ & $b b b$ & $3 b$ & $-\bar{b}$ \\
\hline
\end{tabular}

\section{References}

[1] E. Klempt and J.M. Richard, Rev. Mod. Phys. 82, 1095 (2010), http://dx.doi.org/10.1103 RevModPhys.82.1095

[2] S.K. Bose and L.P.Singh, Phys. Rev.D22, 773 (1980), http://dx.doi.org/10.1103/PhysRevD.22.773
[3] J. Franklin, D.B. Lichtenberg, W. Namgung, and D. Carydas, Phys. Rev. D 24, 2910 (1981), http:// dx.doi.org/10.1103/PhysRevD.24.2910

[4] R.L. Jaffe and J. Kiskis, Phys. Rev. D 13, 1355 (1976), http://dx.doi.org/10.1103/PhysRevD.13.1355

[5] W.A. Ponce, Phys. Rev. D 19, 2197 (1979), http:// dx.doi.org/10.1103/PhysRevD.19.2197

[6] A. Bernotas and V. Simonis, arXiv:1206.6764v2 (2012), http://lanl.arxiv.org/abs/1206.6764v2

[7] A. Bernotas and V. Simonis, Nucl. Phys. A 741, 179 (2004), http://dx.doi.org/10.1016/j.nuclphysa.2004.05.017

[8] A. Halprin and A.K. Kerman, Phys. Rev. D 26, 2532 (1982), http://dx.doi.org/10.1103/ PhysRevD.26.2532

[9] R.J. Johnson and M. Shah-Jahan, Phys. Rev. D 15, 1400 (1977), http://dx.doi.org/10.1103/PhysRevD.15.1400

[10]T. DeGrand, R.L. Jaffe, K. Johnson, and J. Kiskis, Phys. Rev. D 12, 2060 (1975), http://dx.doi. org/10.1103/PhysRevD.12.2060

[11] M.C. Bañuls, I. Scimemi, J. Bernabéu, V. Giménez, and A. Pich, Phys. Rev. D 61, 074007 (2000), http:// dx.doi.org/10.1103/PhysRevD.61.074007

[12] A. Bernotas and V. Simonis, Lith. J. Phys. 48, 127 (2008), http://dx.doi.org/10.3952/lithjphys.48202

[13] W. Roberts and M. Pervin, Int. J. Mod. Phys. A 23, 2817 (2008), http://dx.doi.org/10.1142/ S0217751X08041219

[14]W. Roberts and M. Pervin, Int. I. Mod. Phys. A 24, 2401 (2009), http://dx.doi.org/10.1142/ S0217751X09043407

[15] B. Juliá-Díaz and D.O. Riska, Nucl. Phys. A 739, 69 (2004), http://dx.doi.org/10.1016/j.nuclphysa. 2004.03 .078

[16]N. Barik and M. Das, Phys. Rev. D 28, 2823 (1983), http://dx.doi.org/10.1103/PhysRevD.28.2823

[17]A. Faessler, T. Gutsche, M.A. Ivanov, J.G. Körner, V.E. Lyubovitskij, D. Nicmorus, and K. Pumsa-ard, Phys. Rev. D 73, 094013 (2006), http://dx.doi.org/10.1103/PhysRevD.73.094013

[18]B. Silvestre-Brac, Few-Body Systems 20, 1 (1996), http://dx.doi.org/10.1007/s006010050028

[19] C. Albertus, E. Hernández, J. Nieves and J.M. Verde-Velasco, Eur. Phys. J. A 32, 183 (2007) [Erratumibid. A 36, 119 (2008)], http://dx.doi.org/10.1140 epja/i2007-10364-y

[20]S. Kumar, R. Dhir, and R.C. Verma, J. Phys. G 31, 141 (2005), http://dx.doi.org/10.1088/0954-3899/ $31 / 2 / 006$

[21] R. Dhir and R.C. Verma, Eur. Phys. J. A 42, 243 (2009), http://dx.doi.org/10.1140/epja/i2009-10872-8

[22] B. Patel, A.K. Rai, and P.C. Vinodkumar, J. Phys. G 35, 065001 (2008), http://dx.doi.org/10.1088/0954$3899 / 35 / 6 / 065001$

[23]B. Patel, A. Majethiya, and P.C. Vinodkumar, Pramana 72, 679(2009), http://dx.doi.org/10.1007/ s12043-009-0061-4 
[24] N. Sharma, H. Dahiya, P.K. Chatley, and M. Gupta, Phys. Rev. D 81, 073001 (2010), http://dx.doi. org/10.1103/PhysRevD.81.073001

[25] M.J. Savage, Phys. Lett. B 326, 303 (1994), http:// dx.doi.org/10.1016/0370-2693(94)91326-9

[26] Shi-lin Zhu, W-Y.P. Hwang, and Ze-sen Yang, Phys. Rev. D 56, 7273 (1997), http://dx.doi.org/10.1103/ PhysRevD.56.7273

[27] T.M. Aliev, A. Ozpineci, and M. Savci, Phys. Rev. D 65, 056008 (2002), http://dx.doi.org/10.1103/ PhysRevD.81.073001

[28] T.M. Aliev, K. Azizi, and A. Özpineci, Pys. Rev. D 77, 114006 (2008), http://dx.doi.org/10.1103/ PhysRevD.65.056008
[29]T.M. Aliev, K. Azizi, and A. Özpineci, Nucl. Phys. B 808, 137 (2009), http://dx.doi.org/10.1016/j. nuclphysb.2008.09.018

[30] A. Faessler, T. Gutsche, M.A. Ivanov, J.G. Körner, and V.E. Lyubovitskij, Phys. Rev. D 80, 034025 (2009), http://dx.doi.org/10.1103/ PhysRevD.80.034025

[31] M. Karliner and H.J. Lipkin, Phys. Lett. B 660, 539 (2008), http://dx.doi.org/10.1016/j. physletb.2008.01.023

\title{
SUNKIŲJŲ BARIONŲ MAGNETINIUU MOMENTUU SKAIČIAVIMAS TAIKANT MODIFIKUOTĄ KVARKŲ MAIŞŲ MODELI
}

\author{
A. Bernotas ${ }^{a}$, V. Šimonis ${ }^{b}$ \\ ${ }^{a}$ Vilniaus universiteto Fizikos fakultetas, Vilnius, Lietuva \\ ${ }^{\mathrm{b}}$ Vilniaus universiteto Teorines fizikos ir astronomijos institutas, Vilnius, Lietuva
}

\section{Santrauka}

Suskaičiuoti sunkiųjų barionų, kurių judejjimo kiekio momentai $J=1 / 2$ ir $J=3 / 2$, magnetiniai momentai. Naudotas kvarkų maišų modelis su masių centro judejjimo pataisomis. Tuo atveju, kai pusinio sukinio barionai

yra sudaryti iš skirtingų aromatų kvarkų, būsenų maišymasis dèl hipersmulkiosios sąveikos išnagrinètas smulkiau. Darbo rezultatai palyginami su įvertinimais, gautais naudojant kitus metodus ir modelius. 